\title{
RESONANT FREQUENCY OF CIRCULAR MICROSTRIP ANTENNA BASED ON BPNN
}

\author{
${ }^{1}$ Seyyed Ahmad Mousavi and ${ }^{2}$ Zahra Atlasbaf \\ ${ }^{1}$ Student, Faculty of Electrical and Computer Engineering, Tarbiat Modares University, Iran; \\ ${ }^{2}$ Associate Professor, Faculty of Electrical and Computer Engineering, Tarbiat Modares University, Iran. \\ Email: $\{1$ mousavis@modares.ac.ir, atlasbaf@modares.ac.ir $\}$
}

\begin{abstract}
A Circular Microstrip Antenna (CMA) based on the backpropagation neural network (BPNN) is proposed in this paper. By considering the structure, the antenna has been designed varied dimensions. To obtain resonant frequency; we need electrical parameters and various dimensions with respect to the resonant frequency equation. According to BPNN technique, multi-layer perceptron (MLP) has been used to test the resonant frequency of CMAs and the BPNN is trained using Different learning algorithms and we have compared the results. The network accurately calculates the resonant frequency of CMA.
\end{abstract}

Key Words - Circular Microstrip Antenna (CMA), backpropagation neural network (BPNN), multi-layer perceptron (MLP), artificial neural network (ANN), resonant frequency $\left(\boldsymbol{f}_{r}\right)$

\section{Introduction}

Cost, size, weight, ease of installation, performance, and aerodynamic profile are constraints in satellite, spacecraft, and missile applications so low-profile antennas are required. These antennas are very applicable due to the fact that they are comfortable to planar nonplanar surfaces and they are cheap and simple.[1].

For the microwave communication bands, microstrip antennas are used in high frequency tasks. These antennas have two deficiencies, low bandwidth and low power, which can be addressed by a good design, especially with a suitable choice of dielectric constant, substrate thickness, different patch shapes [2]. In [3] the design of an annular ring microstrip antenna based on ANN is studied and neural network based microstrip antenna design is studied in many papers [4] - [6]. In this paper, we designed a circular microstrip antenna that is based on BPNN. In [7], [8] the ANN is used to analyze microstrip antenna in the form of equilateral triangle and the circular patch antenna respectively. In this study, CST software is employed to obtain the resonant frequency of the suggested antenna. The performance, is calculated from several algorithms and results are tabulated.

A neural network can be considered as a simplified mathematical model of the biological neural network which consists of a collection of interconnected neurons. There is a definition of a neural network [9]: "A neural network is a massively parallel distributed processor that has a natural propensity for storing experiential knowledge and making it available for use. It resembles the brain in two respects: (1) knowledge is acquired by the network through a learning process, and (2) interneuron connection strengths known as synaptic weights are used to store the experiential knowledge."

The art of using artificial neural networks for wireless communication engineering has been achievement momentum. Some of the application of ANN are: microstrip antenna analysis, wideband mobile antenna design, adaptive beamforming, designing of microstrip antenna, CPW patch antenna, direction-of-arrival (DOA) estimation [10]. For the proposed antenna, BPNN method based on the multi-layer perceptron (MLP) has been applied to compute CMA resonant frequencies

\section{Antenna design and Simulation}

A circular patch in microstrip antennas is one of popular patchs as shown in Fig. 1, consisting of a very thin metallic circular slot with the radius $a$ above a substrate having a relative dielectric $\epsilon_{r}$ and height of $h$ on the ground plane.

To design, the proposed antenna, we used CST studio software that is a full-wave software. Respect to the resonant frequency equation of the circular antenna, $f_{r}$ can be changed by varying the substrate height, relative permittivity and patch radius. To calculate the value of $f_{r}, 88$ simulation run out using CST software. Different sizes and dielectric constants for the design are considered and are shown in "Table 1". 
Table 1

CMA parameters

\begin{tabular}{|c|c|c|}
\hline$\epsilon_{r}$ & $h$ & $a$ \\
\hline 2.2 & $0.64,1.1,2.5,3.175$ & $15,18,21,24,27$ \\
\hline 3.55 & $0.64,1.1,2.5,3.175$ & $13,16,1,22,25$ \\
\hline 6.5 & $0.64,1.1,2.5,3.175$ & $10,13,19,19,22$ \\
\hline 9.8 & $0.64,1.1,2.5,3.175$ & $8,11,14,17,20$ \\
\hline
\end{tabular}

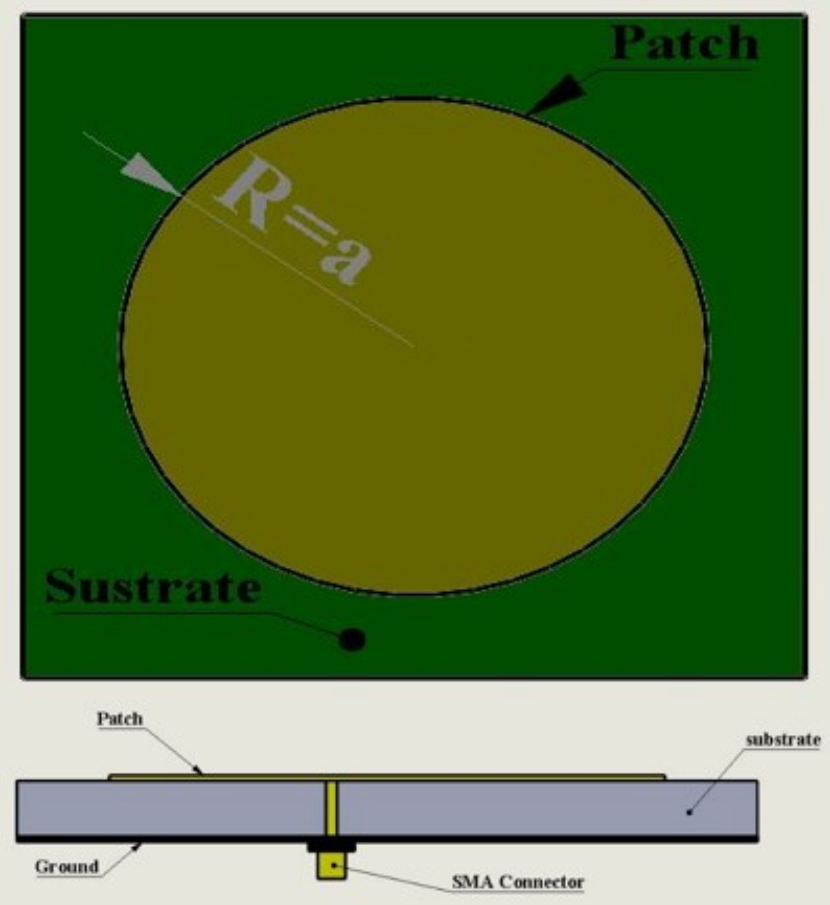

Fig. 1. Geometry of CMA

For example, to obtain $f_{r}$, we simulate first specification of the table1 in CST software as shown in Fig. 2. The parameters that we used $\epsilon_{r}=2.2, h=0.64 \mathrm{~mm}, a=15 \mathrm{~mm}$ and Fig. 2 shows the CMA that design and simulate in CST software. By using these parameters, as shown in Fig. 3, $f_{r}=3.7994$ and the return loss (s11) is equal to -24.696 at this resonant frequency.

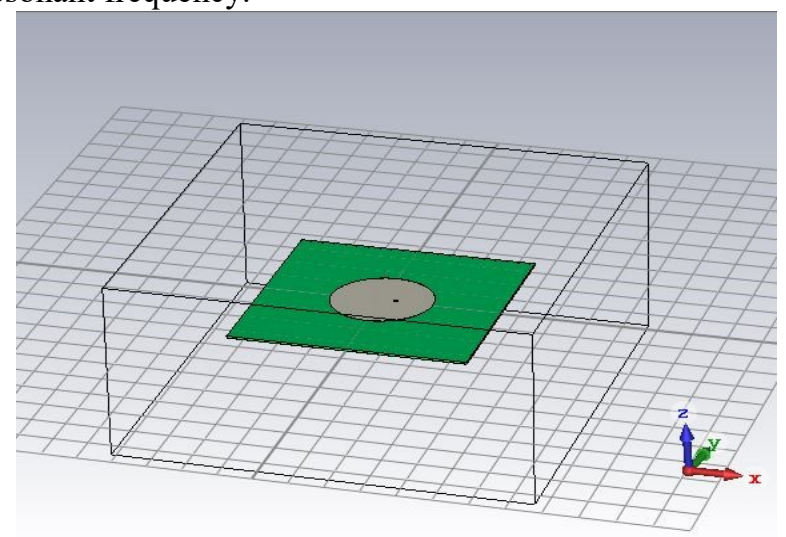

Fig. 2. Structure of CMA by using CST

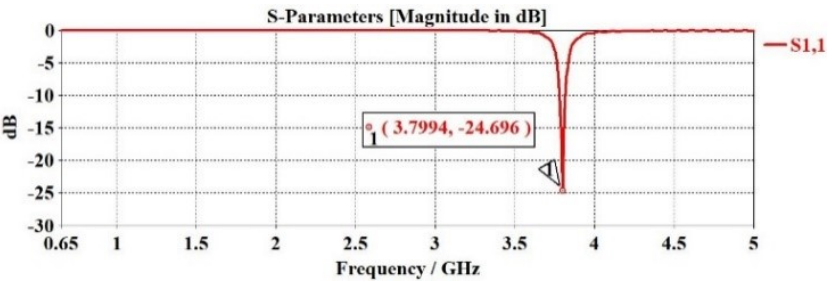

Fig. 3. Return loss

\section{Training and testing of BPNN}

\subsection{Training}

In this section, we give the results of the simulation to BPNN and train it with these data. The advantages of ANN include the ability to learn, the need for low data, and simple implementation. As you can see in Fig. 4, the neural network structure is based on MLP where we give $\epsilon_{r}, h$ and $a$ as inputs to the ANN and the output is the resonant frequency.

The BPNN is one of the most popular neural network algorithms. The BPNN trains the network using an errorbackpropagation algorithm and knows the desired output. In fact, the BPNN is trained by supervised learning.

BPNN's training algorithm consists of two steps, forward and backward, which executes these two steps for each input and output. The target network starts from the output layer and modifies weights. As shown in Fig. 4, our network has three layers, one input layer, one hidden layer and one output layer. The input layer consists of three neurons that receive network inputs. The output layer contains one neuron that gives us the output, resonant frequency, and the hidden layer also contains three neurons. The layers are fully interconnected.

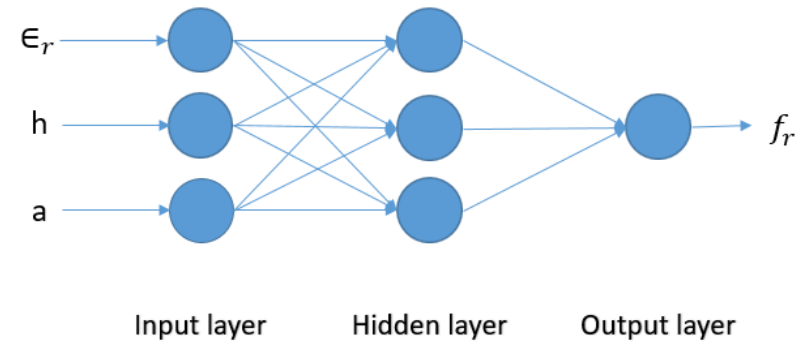

Fig. 4. Diagram of MLP neural network in this study

In Fig. 4, number of neurons in the hidden layer determines the network accuracy. If we increase the number of hidden layer's neurons, the accuracy of the network will rise, but the network will spend more computing time. Several algorithms are used to train the BPNN model. The algorithms that we used are Levenberg-Marquardt backpropagation (LM), Gradient descent backpropagation (GD) and Bayesian regularization (BR).

For proposed antenna 80 values of $\epsilon_{r}, h$ and $a$ are used for training of BPNN model, that these data are obtained with CST software. The training of ANN is done by Matlab. The 
results achieved from ANN by using Matlab are shown in below figures.

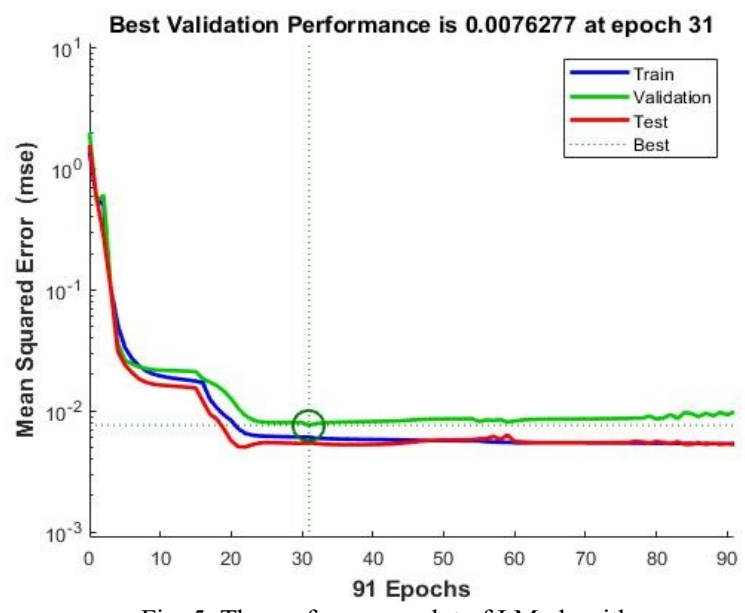

Fig. 5. The performance plot of LM algorithm

Fig. 5 shows MSE plot for LM algorithm. Mean square errors (MSE) can be defined as the average squared difference between the outputs and targets. Lower values are better and also zero means no error. The best validation performance for LM algorithm is 0.0076277 at epoch 31 . Epoch is defined the number of iterations of the algorithm to update the weights.
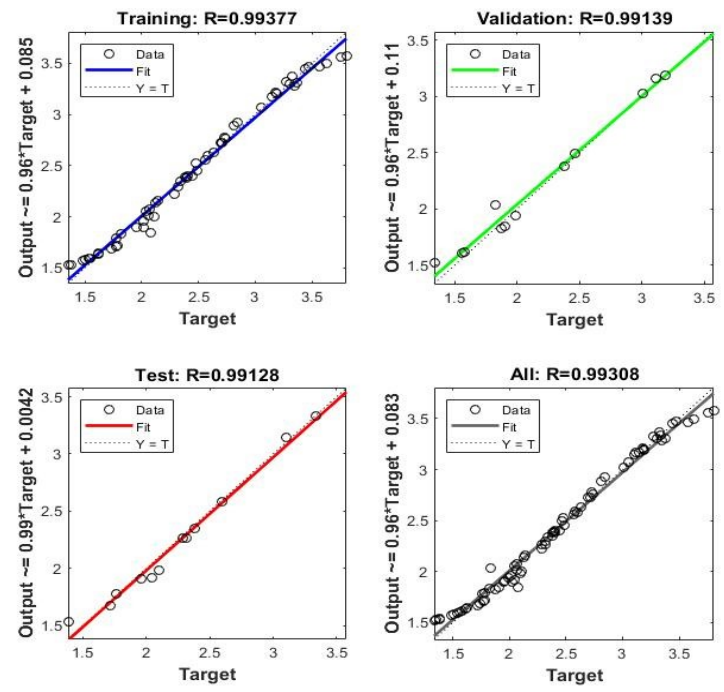

Fig. 6. Regression plot for LM algorithm

Regression $(\mathrm{R})$ values calculate the correlation between outputs and targets. An $\mathrm{R}$ value of 1 means a close relationship, 0 a random relationship. The perfect results between output and target values is displayed by dashed line in the plot. The solid line represents the best output and target fit. The points are called outlier, which are far from the dashed line. The regression achieved is nearly approaching to 1 .

The results of the other algorithms are presented below.

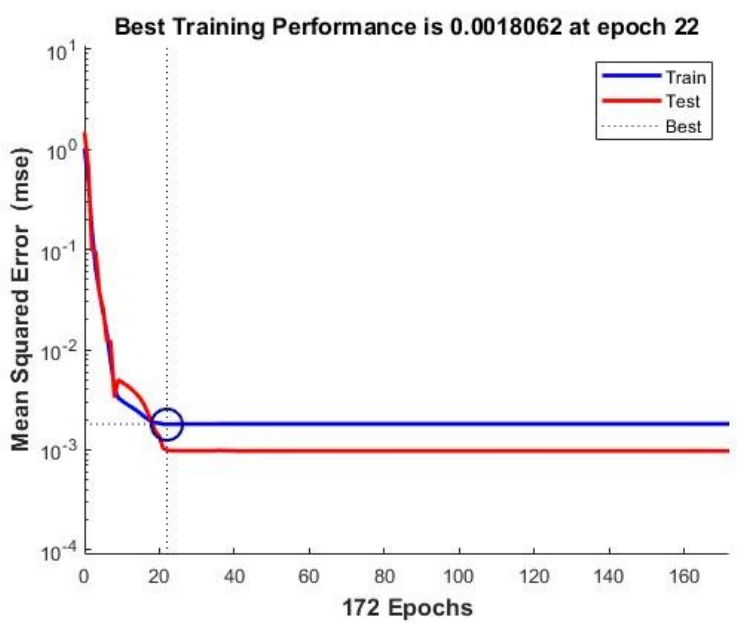

Fig. 7. The performance plot of BR algorithm

Fig. 7 displays MSE plot for Bayesian Regularization algorithm. The best validation training for BR algorithm is 0.0018062 at epoch 22 which is better than the performance of LM algorithm.

Regression (R) values are Plotted in Fig. 8. The plot shows that regression values of BR algorithm is better than LM.
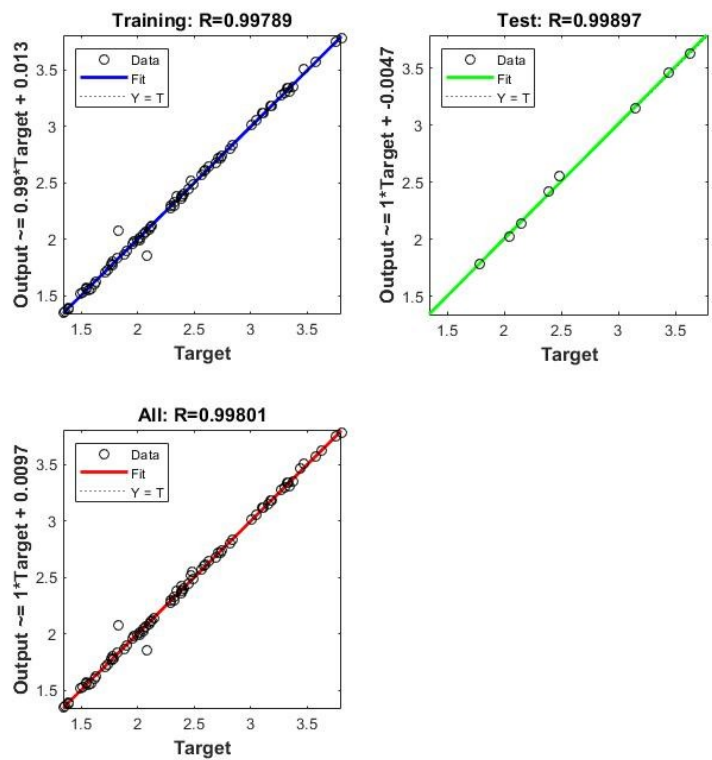

Fig. 8. Regression plot for BR algorithm

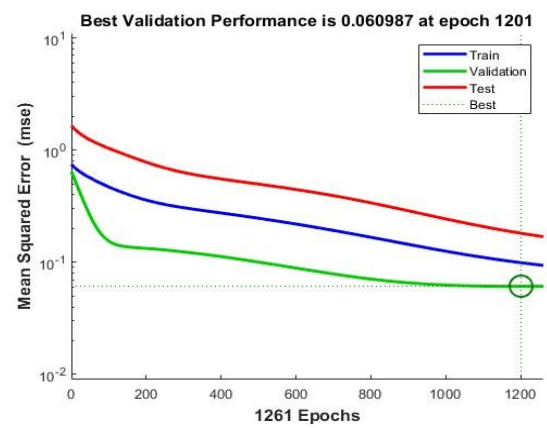

Fig. 9. The performance plot of GD algorithm

JREAS, Vol. 06, Issue 01, Jan 2021 
Fig. 9 and 10 show result of the Gradient Descent algorithm. These figures show that Gradient Descent algorithm has lower precision compared to LM and BR.

Gradient descent is a first-order iterative optimization algorithm to evaluate a function's local minimum.
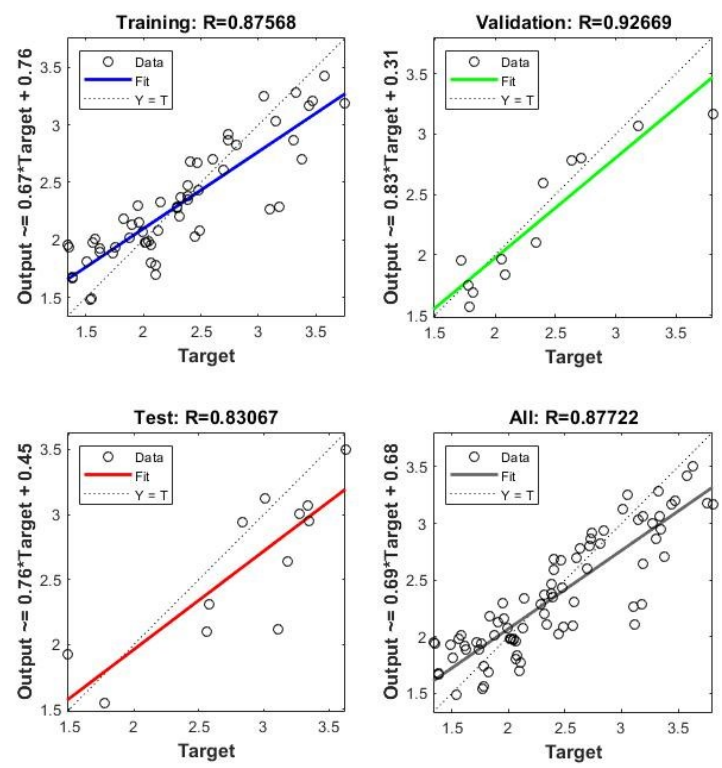

Fig. 10. Regression plot for GD algorithm

\subsection{Testing}

To test and compare our algorithms, we have used 9 data that are listed in Table 2. The simulation results got from HFSS were compared to the BPNN results. The last column shows the resonant frequency obtained from CST.

Table 2

CMA parameters for testing and CST result

\begin{tabular}{|c|c|c|c|c|}
\hline$\#$ & $\epsilon_{r}$ & $\mathrm{~h}$ & $\mathrm{a}$ & $\mathbf{f}_{\text {CST }}$ \\
\hline 1 & 2.2 & 1.57 & 18 & 3.1145 \\
\hline 2 & 2.2 & 1.175 & 30 & 1.8475 \\
\hline 3 & 2.33 & 0.64 & 13 & 4.2393 \\
\hline 4 & 2.33 & 2.5 & 25 & 2.1689 \\
\hline 5 & 2.33 & 1.1 & 25 & 2.228 \\
\hline 6 & 4.5 & 2.5 & 2718 & 2.1823 \\
\hline 7 & 4.5 & 2.5 & 27 & 1.4724 \\
\hline 8 & 9.8 & 0.64 & 5 & 3.9672 \\
\hline 9 & 9.8 & 1.57 & 14 & 1.94 \\
\hline
\end{tabular}

In "Table 3", odd columns show the outputs of artificial neural network using table 2 parameters and even columns display the errors obtained from Equ 1.

$$
\text { Error }=\left|f_{B P N N}-f_{C S T}\right|
$$

Table 3

Comparison of CST and ANN resonant frequency

\begin{tabular}{|c|c|c|c|c|c|c|}
\hline$\#$ & $\mathbf{f}_{\mathbf{L M}}$ & Error $_{\mathbf{L M}}$ & $\mathbf{f}_{\mathbf{B R}}$ & Error $_{\mathbf{B R}}$ & $\mathbf{f}_{\mathbf{G D}}$ & Error $_{\mathbf{G D}}$ \\
\hline 1 & 3.0 .513 & 0.0632 & 3.1151 & 0.0006 & 3.0550 & 0.0595 \\
\hline 2 & 2.0372 & 0.1897 & 1.8328 & 0.0147 & 1.7964 & 0.0511 \\
\hline
\end{tabular}

\begin{tabular}{|l|c|c|c|c|c|c|}
\hline 3 & 4.076 & 0.1633 & 4.2329 & 0.0064 & 3.2351 & 1.0042 \\
\hline 4 & 2.2321 & 0.0632 & 2.1804 & 0.0115 & 1.9946 & 0.1743 \\
\hline 5 & 2.2751 & 0.0471 & 2.2345 & 0.0065 & 2.2614 & 0.0334 \\
\hline 6 & 2.0512 & 0.1311 & 2.1428 & 0.0395 & 2.1260 & 0.0563 \\
\hline 7 & 1.4300 & 0.0424 & 1.5196 & 0.0472 & 1.6392 & 0.1668 \\
\hline 8 & 3.8006 & 0.1666 & 4.2534 & 0.2862 & 2.9754 & 0.9918 \\
\hline 9 & 1.9382 & 0.0018 & 1.9376 & 0.0024 & 2.3622 & 0.4222 \\
\hline
\end{tabular}

Cells that are highlight represent the lowest error rate, the best answer, as it is known that BR algorithm can calculate the resonant frequency with minimal error and has a higher accuracy than the other two algorithms. The table is used to show the accuracy of artificial neural network in some parameters that were not used in the network training process. The duration of the network training lasts less than 1 second, compared to the simulation software, which in addition to the antenna design time, is about 5 minutes requires execution and resonant frequency extraction. This network has a very high speed.

\section{Conclusion}

The research discussed in this paper on the application of the ANN model to obtain the Circular Microstrip Antenna (CMA) resonant frequency. The CST software with 80 different sizes and various dimensions is used to design the antennas. Applied backpropagation algorithm multilayer perceptron's model to train and test the resonant frequencies using different learning algorithms in which Bayesian regularization (BR) give better accuracy compared to others algorithm. The results of CST and ANN model are similar which leads to a better accuracy. So, we showed that the CMA resonant frequencies are similar to ANN results. So, a significant tool in the field of antenna designing and modeling is demonstrated by this ANN. The ANN is simple computing ability, less computing time and less difficulty.

\section{References}

[1] Balanis, Constantine A. Antenna theory: analysis and design. John wiley \& sons, 2016.

[2] Rahman, Saeed Ur, et al. "Analysis of linear antenna array for minimum side lobe level, half power beamwidth, and nulls control using PSO." Journal of Microwaves, Optoelectronics and Electromagnetic Applications 16.2 (2017): 577-591.

[3] Khan, Ijaz, et al. "Design Annular Ring Microstrip Antenna Based on Artificial Neural Network." 2018 2nd IEEE Advanced Information Management, Communicates, Electronic and Automation Control Conference (IMCEC). IEEE, 2018.

[4] Türker, Nurhan, Filiz Güneş, and Tülay Yildirim. "Artificial neural design of microstrip antennas." Turkish Journal of Electrical Engineering \& Computer Sciences 14.3 (2007): 445-453.

[5] Tighilt, Yamina, Farid Bouttout, and Abdelhafid Khellaf. "Modeling and design of printed antennas using neural networks." International Journal of RF 
and Microwave Computer-Aided Engineering 21.2 (2011): 228-233.

[6] Singh, Bablu Kumar. "Design of rectangular microstrip patch antenna based on Artificial Neural Network algorithm." 2015 2nd International Conference on Signal Processing and Integrated Networks (SPIN). IEEE, 2015.

[7] S. S. and K. Guney, "Calculation of resonant frequency for an equilateral triangular microstrip antenna using artificial neural Networks," Microw. Opt. Technol. Lett., vol. 14, no. 2, pp. 89-93, 1996.
[8] F. Abboud, J. P. Damiano, and A. Papiernik, "A new model for calculating the input impedance of coaxfed circular microstrip antennas with and without air gaps," IEEE Trans. Antennas Propag., vol. 38, no. 11, pp. 1882-1885, 1990.

[9] Haykin, Simon. Neural networks: a comprehensive foundation. Prentice Hall PTR, 1994.

[10] Patnaik, Amalendu, et al. "Applications of neural networks in wireless communications." IEEE Antennas and Propagation Magazine 46.3 (2004): 130137. 